Pacific Journal of Mathematics

ON SOLUTIONS OF DIFFERENTIAL EQUATIONS WHICh 


\section{ON SOLUTIONS OF DIFFERENTIAL EQUATIONS WHICH SATISFY CERTAIN ALGEBRAIC RELATIONS}

\section{SteVen SPERBER}

In the following, we provide another proof (Theorem 3.1 below) of recent results of Harris-Sibuya, using some elementary commutative algebra. Our purpose is to give a uniform treatment for their results which also permits some generalization. We note that the study of differential equations under the hypothesis that the solutions satisfy an algebraic relation is not new. Fano, among others, made a systematic study of this situation in the last century. Also Lamé equations in which two solutions have a rational function as their product have proved to be a good source of examples for unusual arithmetic behavior. But in the case of Harris-Sibuya, as well as the present paper, the solutions need not be solutions of the same linear equation. In the treatment below the differential equation only enters in dilineating a type of recursion.

1. Let $R=K\left[\left\{\Lambda_{i}\right\}_{i=1}^{N}\right.$ be a polynomial ring over a field $K$. Assume that it is graded by assigning to each variable $\Lambda_{i}$ a natural number, $w\left(\Lambda_{l}\right)=w_{i} \in \mathbf{N}$, and then assigning

$$
w\left(\prod_{i=1}^{N} \Lambda_{i}^{\mu_{i}}\right)=\sum_{i=1}^{N} \mu_{i} w_{i} .
$$

The following result makes use of some elementary commutative algebra.

(1.1) THEOREM. Let $I$ be the ideal of $R$ generated by a collection of polynomials, $\left\{f_{\beta}(\Lambda)\right\}_{\beta \in \Gamma} \subseteq R$. Let $\tilde{f}_{\beta}(\Lambda)$ be the leading homogeneous form of $f_{\beta}(\Lambda)$ with respect to the above grading; let $J$ be the homogeneous ideal

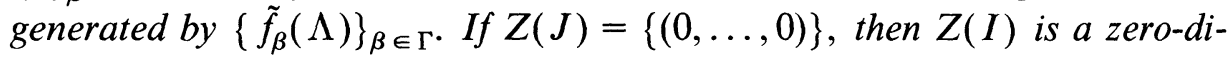
mensional variety.

Proof. Since $Z(J)=\{(0, \ldots, 0)\}$, the Nullstellansatz implies that $\sqrt{J}$, the radical of $J$, satisfies

$$
\sqrt{J}=\left(\Lambda_{1}, \ldots, \Lambda_{N}\right) .
$$

Thus, for each $i \in\{1,2, \ldots, N\}$, there exists an $m_{i} \in \mathbf{N}$ such that $\Lambda_{i}^{m_{t}} \in J$. The sequence $\left\{\Lambda_{1}^{m_{1}}, \ldots, \Lambda_{N}^{m_{N}}\right\}$ forms a regular sequence (in any order). Let 
us denote $\tilde{h}_{i}(\Lambda)=\Lambda_{i}^{m_{t}}$ and write

$$
\tilde{h}_{i}(\Lambda)=\sum_{\beta \in \Gamma} \tilde{A}_{i, \beta}(\Lambda) \tilde{f}_{\beta}(\Lambda)
$$

where $\tilde{A}_{i, \beta}(\Lambda)$ is homogeneous of weight equal to $m_{i} w_{l}-w\left(\tilde{f}_{\beta}\right)$.

Define

$$
h_{i}(\Lambda)=\sum_{\beta \in \Gamma} \tilde{A}_{i, \beta}(\Lambda) f_{\beta}(\Lambda)
$$

Clearly the leading form of $h_{i}(\Lambda)$ is $\tilde{h}_{i}(\Lambda)$ so that

$$
h_{i}(\Lambda)=\tilde{h}_{i}(\Lambda)+\hat{h}_{i}(\Lambda)
$$

with $w\left(\hat{h}_{i}(\Lambda)\right)<m_{i} w_{i}$. We assert that $\left\{h_{1}(\Lambda), \ldots, h_{N}(\Lambda)\right\}$ is a regular sequence (in any order). Suppose

$$
\sum_{i=1}^{k} \xi_{i}(\Lambda) h_{i}(\Lambda)=0 .
$$

Let $w$ be the weight of the highest weighted terms that appear on the left-side (before cancellation). We write

$$
\xi_{i}(\Lambda)=\tilde{\xi}_{i}(\Lambda)+\hat{\xi}_{i}(\Lambda)
$$

where $w\left(\xi_{i}\right)=w\left(\tilde{\xi}_{i}\right)=w-m_{i} w_{i}>w\left(\hat{\xi}_{i}\right)$. We claim there exists a skewsymmetric set $\alpha_{\imath j}(\Lambda) \in R$ such that

$$
\xi_{i}(\Lambda)=\sum_{j=1}^{k} \alpha_{i j}(\Lambda) h_{j}(\Lambda)
$$

for each $i, 1 \leq i \leq k$. We proceed by induction on $w$. Since

$$
\sum_{i=1}^{k} \tilde{\xi}_{i} \tilde{h}_{i}=0
$$

there is a skew-symmetric set of homogeneous polynomials $\left\{\tilde{\eta}_{i j}\right\}_{i \leq i, j \leq k}$ $\subseteq R$ such that

$$
\begin{gathered}
\tilde{\xi}_{i}(\Lambda)=\sum_{j=1}^{k} \tilde{\eta}_{i j}(\Lambda) \tilde{h}_{j}(\Lambda), \\
w\left(\tilde{\eta}_{i j}(\Lambda)\right)=w-m_{\imath} w_{l}-m_{J} w_{j} .
\end{gathered}
$$

We may rewrite

$$
\tilde{\xi}_{i}(\Lambda)=\sum_{j=1}^{k} \tilde{\eta}_{i j} h_{j}-\sum_{j=1}^{k} \tilde{\eta}_{i j} \hat{h}_{j}
$$


so that

$$
0=\sum_{i=1}^{k} \xi_{i} h_{i}=\sum_{i=1}^{k} \tilde{\xi}_{i} h_{i}+\sum_{i=1}^{k} \hat{\xi}_{i} h_{i}=\sum_{i=1}^{k}\left(\hat{\xi}_{i}-\sum_{j=1}^{k} \tilde{\eta}_{i j} \hat{h}_{j}\right) h_{i} .
$$

If we write,

$$
\zeta_{i}=\hat{\xi}_{i}-\sum_{j=1}^{k} \tilde{\eta}_{i j} \hat{h}_{j}
$$

then $w\left(\zeta_{i}\right)<w\left(\xi_{i}\right)$ so that in

$$
\sum_{i=1}^{k} \zeta_{i} h_{i}=0
$$

the highest weight term appearing on the left is strictly less than $w$. By induction

$$
\zeta_{i}=\hat{\xi}_{i}-\sum_{j=1}^{k} \tilde{\eta}_{i j} \hat{h}_{j}=\sum_{j=1}^{k} \gamma_{i j} h_{j}
$$

so that

$$
\hat{\xi}_{i}=\sum_{j=1}^{k} \gamma_{i j} h_{j}+\sum_{j=1}^{k} \tilde{\eta}_{i j} \hat{h}_{j}
$$

and

$$
\xi_{i}=\sum_{j=1}^{k}\left(\tilde{\eta}_{i j}+\gamma_{i j}\right) h_{j}
$$

as required.

In a similar manner, we can show that

$$
\left(h_{1}(\Lambda), \ldots, h_{N}(\Lambda)\right) \neq R .
$$

For suppose a relation $1=\sum_{i=1}^{N} \alpha_{i} h_{i}$ exists with $\alpha_{i} \in R$ and $M=$ $\max _{1 \leq i \leq N}\left\{w\left(\alpha_{i}\right)+m_{i} w_{i}\right\}$. As before we write $\alpha_{i}=\tilde{\alpha}_{i}+\hat{\alpha}_{i}$ with $w\left(\alpha_{i}\right)=$ $w\left(\tilde{\alpha}_{t}\right)>w\left(\hat{\alpha}_{i}\right)$ and $\tilde{\alpha}_{i}$ homogeneous. We may assume the $\alpha_{i}$ 's chosen so that $M$ is minimal. Clearly $\sum_{i=1}^{N} \tilde{\alpha}_{i} \tilde{h}_{i}=0$ where $\tilde{\alpha}_{i}(\Lambda)$ is the leading form of $\alpha_{t}(\Lambda)$. This implies the existence of a skew-symmetric set of homogeneous polynomials $\left\{\tilde{\gamma}_{i j}\right\}_{i, j=1}^{N} \subseteq R$ satisfying

$$
\begin{gathered}
\tilde{\alpha}_{i}=\sum_{j=1}^{N} \tilde{\gamma}_{i j} \tilde{h}_{j}=\sum_{j=1}^{N} \tilde{\gamma}_{i j} h_{j}-\sum_{j=1}^{N} \tilde{\gamma}_{i j} \hat{h}_{J}, \\
w\left(\tilde{\gamma}_{i j}\right)=w\left(\alpha_{i}\right)-w\left(\tilde{h}_{j}\right) .
\end{gathered}
$$


Using this

$$
1=\sum_{i=1}^{N}\left(\hat{\alpha}_{\imath}-\sum_{j=1}^{N} \tilde{\gamma}_{\imath j} \hat{h}_{J}\right) h_{i}
$$

and

$$
\max _{1 \leq l \leq N}\left\{w\left(\hat{\alpha}_{i}-\sum_{j=1}^{N} \tilde{\gamma}_{i j} \hat{h}_{j}\right)+m_{i} w_{l}\right\}<M .
$$

This contradicts the choice of $\left\{\alpha_{l}\right\}_{i=1}^{N} \subseteq R$.

The point of this is to establish that

$$
\operatorname{depth}(I) \stackrel{\dot{ }}{=} N
$$

so that, since we are in a Cohen-Macauley ring,

$$
\operatorname{height}(I)=\operatorname{depth}(I)=N \text {. }
$$

Since $I$ is an ideal in a polynomial ring, this implies that dimension $(I)=0$ which completes the proof of the theorem.

2. We now apply this algebraic result to differential equations. Let $K$ be a field of characteristic zero.

(2.1) Definition. Let $\left(N_{1}, \ldots, N_{r}\right)$ be an $r$-tuple of non-negative integers. Let $F$ be a field extension of $K$. A polynomial $G\left(Y_{1}, \ldots, Y_{r}\right) \in$ $K\left[Y_{1}, Y_{2}, \ldots, Y_{r}\right]$ is called $\left(N_{1}, \ldots, N_{r}\right)$-PF ("polynomial-free") over $F$ provided that $G\left(y_{1}, \ldots, y_{r}\right)=0$ with $y_{i} \in F[t]$, ( $t$ transcendental over $F), \operatorname{deg}_{t}\left(y_{l}\right) \leq N_{i}$, implies $y_{i} \in F$.

(2.2) Examples. (i) $Y_{1}^{m_{1}} Y_{2}^{m_{2}} \ldots Y_{r}^{m_{r}}=1 ; m_{i} \geq 1$ is $\left(N_{1}, \ldots, N_{r}\right)$-PF over $F$ for arbitrary $\left(N_{1}, \ldots, N_{r}\right) \in\left(\mathbf{Z}_{\geq 0}\right)^{r}, F / K$.

(ii) $Y_{1}=Y_{2}^{m_{2}} Y_{3}^{m_{3}} \cdots Y_{r}^{m_{r}} ; m_{l} \geq 1$ is $\left(N_{1}, \ldots, N_{r}\right)$-PF over $F$ provided $N_{1}<\min \left(m_{2}, m_{3}, \ldots, m_{r}\right)$.

In both of these examples, the verification is a trivial observation concerning degrees. Other examples can easily be constructed.

(2.3) Theorem. Assume for each $i \in\{1,2, \ldots, r\}$,

$$
y_{i}(t)=\sum_{j=1}^{\infty} \lambda_{j}^{(l)} t^{\jmath} \in F[[t]]
$$

is a formal power series solution of a differential equation $L_{l} y_{l}=0$ where $L_{l} \in K[[t]][d / d t]$ is a linear differential operator of order $N_{l}+1$ with an 
ordinary point at $t=0$. Assume also that $G\left(y_{1}, \ldots, y_{r}\right)=0$ where $G(Y) \in$ $K\left[Y_{1}, \ldots, Y_{r}\right]$ is $\left(N_{1}, \ldots, N_{r}\right)$-PF over $\bar{K}$, where $\bar{K}$ is the algebraic closure of $K\left(\left\{\lambda_{0}^{(i)}\right\}_{i=1}^{r}\right)$. Then $\lambda_{j}^{(i)} \in \bar{K}$ for all $i$ and all $j$.

Proof. Replacing $K$ by $K\left(\left\{\lambda_{0}^{(i)}\right\}_{i=1}^{r}\right)$ if necessary, we may assume $\left\{\lambda_{0}^{(i)}\right\}_{i=1}^{r} \subseteq K$ and are fixed. Let $\left\{\Lambda_{j}^{(i)}\right\}_{1 \leq i \leq r ; j \geq 1}$ be algebraically independent over $K(t)$. Let $Z_{i}=\lambda_{0}^{(i)}+\sum_{j=1}^{\infty} \Lambda_{j}^{(i)} t^{j}$. We view $\left\{\lambda_{j}^{(i)}\right\}_{1 \leq i \leq r ; j \geq 1}$ as a solution of the system of polynomials

$$
\left\{\tilde{f}_{k}\left(\left\{\Lambda_{j}^{(i)}\right\}_{j \geq 1 ; 1 \leq i \leq r}\right) \in \tilde{R}=K_{0}\left[\left\{\Lambda_{j}^{(i)}\right\}_{j \geq 1 ; 1 \leq i \leq r}\right]\right\}_{k \geq 1}
$$

where these polynomials are defined as follows

$$
G\left(Z_{1}, \ldots, Z_{r}\right)=G\left(\lambda_{0}^{(i)}, \ldots, \lambda_{0}^{(r)}\right)+\sum_{k=1}^{\infty} \tilde{f}_{k}\left(\left\{\Lambda_{j}^{(i)}\right\}\right) t^{k}
$$

(Of course, $G\left(\lambda_{0}^{(1)}, \ldots, \lambda_{0}^{(r)}\right)=0$ ). The hypothesis that $y_{i}(t)$ satisfies a linear differential equation of order $N_{i}+1$ with coefficients in $K[[t]]$ and an ordinary point at $t=0$ means the $\left\{\lambda_{j}^{(i)}\right\}_{j \geq 1}$ satisfy a recursion of the form

$$
\Lambda_{N_{i}+l}^{(i)}=\gamma_{l}^{(i)}\left(\lambda_{0}^{(i)}, \Lambda_{1}^{(i)}, \ldots, \Lambda_{N_{i}}^{i}\right) ; \quad l \geq 1
$$

where $\gamma_{i}^{(i)}$ is a linear form in $N_{i}+1$ variables defined over $K$.

Substituting (2.5) into (2.4), $\lambda=\left(\left\{\lambda_{j}^{(i)}\right\} \mid 1 \leq j \leq N_{i} ; 1 \leq i \leq r\right)$ satisfies an infinite system of polynomials in a finite number $N=\sum_{i=1}^{r} N_{i}$ variables:

$$
F_{k}(\Lambda) \in R=K_{0}\left[\left\{\Lambda_{j}^{(i)}\right\} \mid 1 \leq j \leq N_{i} ; 1 \leq i \leq r\right], \quad k \geq 1 .
$$

In particular $\lambda$ is a point on the algebraic variety $Z(I)$ where $I \subseteq R$ is the ideal generated by the $F_{k}(\Lambda), k \geq 1$. It suffices then to show $Z(I)$ is zero dimensional.

We give $R$ and $\tilde{R}$ a grading by assigning $w\left(\Lambda_{j}^{(i)}\right)=j$, for all $i$, $1 \leq i \leq r$, and all $j, j \geq 1$. Then $\tilde{f}_{k}$ is a homogeneous polynomial of weight $k$. Let $\bar{f}_{k}(\Lambda) \in R$ be the image of $F_{k}(\Lambda)$ under the ring-homomorphism $\varphi: \tilde{R} \rightarrow R$ defined by sending $\Lambda_{j}^{(i)} \rightarrow 0$, whenever $j \geq N_{i}+1$ and $\Lambda_{j}^{(i)} \rightarrow \Lambda_{j}^{(i)}$ whenever $j \leq N_{i}$. Then, since the substitution (2.5) involves replacing $\Lambda_{N_{1}+l}^{(i)}$ by a polynomial of strictly smaller weight, it follows that $\bar{f}_{k}(\Lambda) \in J$ where $J$ is the homogeneous ideal generated by the leading forms $\tilde{F}_{k}(\Lambda)$ of the polynomials $F_{k}(\Lambda),(2.6)$.

Since

$$
\varphi G\left(Z_{1}, \ldots, Z_{r}\right)=G\left(\varphi Z_{1}, \ldots, \varphi Z_{r}\right)=\sum_{k=1}^{\infty} \bar{f}_{k}(\Lambda) t^{k}
$$


the hypothesis that $G$ is $\left(N_{1}, \ldots, N_{r}\right)$ - PF over $\bar{K}$, the algebraic closure of $K$, implies that

$$
Z(\tilde{J})=\{(0, \ldots, 0)\}
$$

where $\tilde{J}$ is the ideal of $R$ generated by the $\bar{f}_{k}(\Lambda)$. Clearly then $Z(J)=$ $\{(0, \ldots, 0)\}$ and $Z(I)$ is zero-dimensional.

3. As in the case of Harris-Sibuya [3], [4], we utilize a homomorphism of differential rings to obtain our main result.

(3.1) TheOREM. Let $K$ be a differential field of characteristic zero. Let $F / K$ be an extension of differential fields. Assume for each $i, 1 \leq i \leq r$, $y_{\imath} \in F$ is annihilated by a linear differential operator $L_{\imath} \in K[D]$,

$$
L_{i}=\sum_{j=0}^{N_{t}+1} \alpha_{i j} D^{j}, \quad \alpha_{i j} \in K
$$

order $L_{i}=N_{i}+1$. Assume also that

$$
y_{1}=y_{2}^{m_{2}} y_{3}^{m_{3}} \cdots y_{r}^{m_{r}}
$$

where $N_{1}+1 \leq \min \left\{m_{2}, \ldots, m_{r}\right\}$. Then $D y_{i} / y_{\imath}$ is algebraic over $K$, for each $i, 1 \leq i \leq r$.

Remarks. (i) The result above includes both Harris-Sibuya theorems as special cases. In particular, the case $y_{1} y_{2}=1$ is the case $r=3$, $m_{2}=m_{3}=1, y_{1}=1$.

(ii) We are indebted to $M$. Singer for pointing out that the case $y_{1} y_{2}=1$ follows immediately from a result of $\mathrm{S}$. Morrison which appears in [1, Theorem 3].

Proof of Theorem. The map

$$
\theta: F \rightarrow F[[t]]
$$

defined by

$$
\theta(\alpha)=\sum_{l=0}^{\infty} \frac{D^{l} \alpha}{l !} t^{l}
$$

has been studied by Robba [5]. It is an injective homomorphism of differential rings:

$$
\theta(D \alpha)=\frac{d}{d t} \theta(\alpha)
$$


Since $y_{i}$ satisfies an $\left(N_{i}+1\right)$ st order linear differential equation defined over $K$, it follows easily that $\theta\left(y_{i}\right) \in F[[t]]$ is a power-series solution of an $\left(N_{t}+1\right)$-st order linear differential equation with coefficients in $K[[t]]$ having an ordinary point at $t=0$. Since $y_{i} \in F$ is a constant with respect to the derivation $d / d t$ of $F[[t]]$ it follows that

$$
u_{i}=\theta\left(y_{i}\right) / y_{i}=1+\sum_{l=1}^{\infty} \frac{D^{l}\left(y_{i}\right)}{l ! y_{i}} t^{l}
$$

is also a solution of an $\left(N_{i}+1\right)$-st order linear differential equation with coefficients in $K[[t]]$ having an ordinary point at $t=0$. In addition, the relation $y_{1}=y_{2}^{m_{2}}, \ldots, y_{r}^{m_{r}}$ yields $\theta\left(y_{1}\right)=\theta\left(y_{2}\right)^{m_{2}} \cdots \theta\left(y_{r}\right)^{m_{r}}$ and therefore $u_{1}=u_{2}^{m_{2}} \cdots u_{r}^{m_{r}}$. As a consequence of the previous section, the coefficients of the $u_{i}$ are algebraic over $K$ as required.

4. The method above has wider applications than those given above although in most cases the conclusions that can be immediately drawn are significantly weaker than the conclusions of Harris-Sibuya.

(4.1) TheOREM. Let $K$ be a differential field of characteristic zero. Let $F / K$ be an extension of differential fields. Assume for each $i=1,2, y_{i} \in F$ is annihilated by a linear differential operator $L_{l} \in K[D]$ of order $N_{i}+1$. Let $K_{0}=$ constants of $K=\{\alpha \in K \mid D(\alpha)=0\}$. Assume that $G\left(y_{1}, y_{2}\right)=0$ where $G\left(Y_{1}, Y_{2}\right) \in K_{0}\left[Y_{1}, Y_{2}\right]$ is $\left(N_{1}, N_{2}\right)-P F$ over $\bar{K}$, the algebraic closure of $K$. Then $y_{i}$ satisfies a first-order algebraic differential equation with coefficients in $K$.

Proof. We proceed as in (3.1) working with $\theta\left(y_{i}\right)=\sum_{j=0}^{\infty} \lambda_{j}^{(i)} t^{j}$ (with $\lambda_{j}^{(l)}=D^{j}\left(y_{i}\right) / j$ !) instead of $\theta\left(y_{i}\right) / y_{i}$. The conclusion in this case is that the coefficients of $\theta\left(y_{i}\right)$ (including $\lambda_{1}^{(i)}=D y_{i}$ ) are algebraic over $K\left(\lambda_{0}^{(1)}, \lambda_{0}^{(2)}\right)=K\left(y_{1}, y_{2}\right)$. Since $y_{1}$ and $y_{2}$ are algebraically dependent, the desired conclusion follows.

It is clear that in the above treatment, the role played by the linear differential operator is to provide recursions (as in (2.5)) having the properties that they are polynomial in nature and they reduce the given weight (the weight being dictated by the algebraic relation satisfied by the solutions). In particular, no special use was made of the linearity of the recursion in (2.5).

(4.2) Definition. Let $\mathscr{K}$ be a differential ring. Let $\mathscr{F}$ be a differential extension ring of $\mathscr{K}$. We say $y \in \mathscr{F}$ is weighted monic over $\mathscr{K}$ if $y$ 
satisfies an algebraic differential equation of the following type

$$
y^{(n)}=H\left(y, \ldots, y^{(n-1)}\right)
$$

where $H\left(z_{1}, \ldots, z_{n}\right) \in \mathscr{K}\left[z_{1}, \ldots, z_{n}\right]$ and where the monomials $z^{\alpha}=$ $z_{1}^{\alpha_{1}} \cdots z_{n}^{\alpha_{n}}$ of $H$ satisfy

$$
\sum_{i=1}^{n}(i-1) \alpha_{i}<n
$$

The importance for us of this definition is that when $y$ is weighted monic over $K$ then $\theta(y)=\sum_{k=0}^{\infty} \lambda_{k} t^{k}$ is a power series in $t$ which is weighted monic over $K[[t]]$ and the recursion satisfied by the coefficients has the form

$$
\lambda_{k+n}=Q_{k}\left(\lambda_{0}, \lambda_{1}, \ldots, \lambda_{n-1}\right)
$$

where $Q_{k} \in K\left[\Lambda_{0}, \Lambda_{1}, \ldots, \Lambda_{n-1}\right]$, and if $\Lambda^{\beta}=\Lambda_{0}^{\beta_{0}} \Lambda_{1}^{\beta_{1}} \cdots \Lambda_{n}^{\beta_{n}}$ is a monomial of $Q_{k}$ then

$$
w\left(\Lambda_{0}^{\beta_{0}} \Lambda_{1}^{\beta_{1}}, \ldots, \Lambda_{n}^{\beta_{n}}\right)<k+n .
$$

(4.3) REMARK. It follows from the above discussion that the hypothesis of (3.1) and (4.1) that $y_{\imath}$ satisfies a linear differential equation over $K$ can be replaced by the weaker hypothesis that the $y_{t}$ are weighted monic over $K$.

Finally, it is not difficult to extend the above results to solutions of certain overdetermined systems of partial differential equations.

We thank W. A. Harris, Jr. and Y. Sibuya, for their encouragement.

\section{REFERENCES}

[1] P. Blum, Rational functions on differentially closed sets, Amer. J. Math., XCIV, no. 3 (1972), 676-684.

[2] G. Fano, Ueber lineare homogene Differentialgleichungen wit algebraischen Relationen zwischen den Fundamentalosungen, Math. Ann., Bd 53 (1900), 493-590.

[3] W. A. Harris, Jr., and Y. Sibuya, The reciprocals of solutions of linear ordinary differential equations, IMA preprint \#72, to appear Adv. in Math.

[4] _ The $n$-th roots of solutions of linear ordinary differential equations, in preparation. (Abstract AMS Meeting, Anaheim, Jan. 1985), to appear Proc. Amer. Math. Soc.

[5] P. Robba, Nouveau point de vue sur le prolongement algébrique, Groupe d'étude $d$ 'analyse ultrametrique, 4 annee, (1976/77), no. 5.

Received March 29, 1985. 


\section{PACIFIC JOURNAL OF MATHEMATICS EDITORS}

\author{
V. S. VARADARAJAN \\ (Managing Editor) \\ University of California \\ Los Angeles, CA 90024 \\ HeRbert Clemens \\ University of Utah \\ Salt Lake City, UT 84112 \\ R. FINN \\ Stanford University \\ Stanford, CA 94305
}

HERMANN FLASCHKA

University of Arizona

Tucson, AZ 85721

RAMESH A. GANGOLLI

University of Washington

Seattle, WA 98195

VAUGHAN F. R. JONES

University of California

Berkeley, CA 94720

ROBION KIRBY

University of California

Berkeley, CA 94720
C. C. MOORE

University of California

Berkeley, CA 94720

H. SAMELSON

Stanford University

Stanford, CA 94305

HAROLD STARK

University of California, San Diego

La Jolla, CA 92093

\section{ASSOCIATE EDITORS}
R. Arens
E. F. BECKENBACH
B. H. NEUMANN
F. WOLF
K. YoshIDA (1906-1982)

\section{SUPPORTING INSTITUTIONS}

UNIVERSITY OF ARIZONA

UNIVERSITY OF BRITISH COLUMBIA

CALIFORNIA INSTITUTE OF TECHNOLOGY

UNIVERSITY OF CALIFORNIA

MONTANA STATE UNIVERSITY

UNIVERSITY OF NEVADA, RENO

NEW MEXICO STATE UNIVERSITY

OREGON STATE UNIVERSITY
UNIVERSITY OF OREGON

UNIVERSITY OF SOUTHERN CALIFORNIA

TANFORD UNIVERSITY

UNIVERSITY OF HAWAII

UNIVERSITY OF TOKYO

UNIVERSITY OF UTAH

WASHINGTON STATE UNIVERSITY

UNIVERSITY OF WASHINGTON 


\section{Pacific Journal of Mathematics}

Vol. 124, No. $1 \quad$ May, 1986

Kinetsu Abe and Martin Andrew Magid, Relative nullity foliations and indefinite isometric immersions

Erik P. van den Ban, A convexity theorem for semisimple symmetric

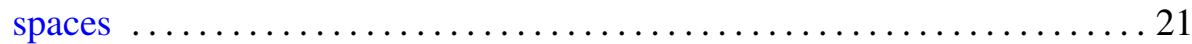

Bo Berndtsson and Thomas Joseph Ransford, Analytic multifunctions, the $\bar{\partial}$-equation, and a proof of the corona theorem .................. 57

Brian Boe and David H. Collingwood, Intertwining operators between

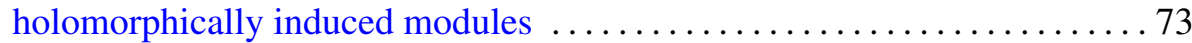

Giuseppe Ceresa and Alessandro Verra, The Abel-Jacobi isomorphism for

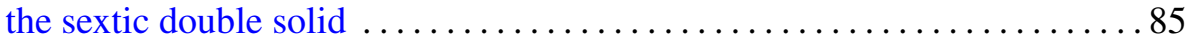

Kun Soo Chang, Jae Moon Ahn and Joo Sup Chang, An evaluation of the conditional Yeh-Wiener integral ........................... 107

Charles Dale Frohman, Minimal surfaces and Heegaard splittings of the

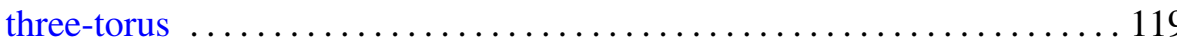

Robert M. Guralnick, Power cancellation of modules $\ldots \ldots \ldots \ldots \ldots \ldots 131$

Kenneth Hardy and Kenneth S. Williams, On the solvability of the Diophantine equation $d V^{2}-2 e V W-d W^{2}=1 \ldots \ldots \ldots \ldots \ldots \ldots \ldots \ldots \ldots$

Ray Alden Kunze and Stephen Scheinberg, Alternative algebras having

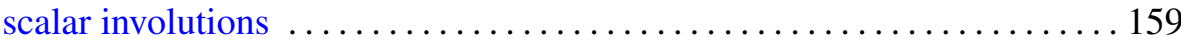

W. B. Raymond Lickorish and Kenneth Millett, The reversing result for the Jones polynomial $\ldots \ldots \ldots \ldots \ldots \ldots \ldots \ldots \ldots$

Guido Lupacciolu, A theorem on holomorphic extension of CR-functions

William Schumacher Massey and Lorenzo Traldi, On a conjecture of K. Murasugi

Dinakar Ramakrishnan, Spectral decomposition of $L^{2}(N \backslash \mathrm{GL}(2), \eta)$

Steven L. Sperber, On solutions of differential equations which satisfy certain algebraic relations 